\title{
A sparse sampling strategy for angular superresolution of real beam scanning radar
}

\author{
Yin Zhang ${ }^{*}{ }^{\dagger}$, Junjie $\mathrm{Wu}^{\dagger}$ and Jianyu Yang ${ }^{\dagger}$
}

\begin{abstract}
This paper investigates techniques for angular superresolution using limited data of real beam scanning radar (RBSR). In order to improve the angular resolution of RBSR, many algorithms have been proposed. However, for most algorithms, large amounts of sampling data is necessary. The requirement of data increases the burden of the radar system. Fortunately, the sparse signal reconstruction techniques provide a new train of thought for us. It has been proved in array signal processing and image processing that the techniques only need limited sampling data to realize DOA estimation and image superresolution. This paper describes the sparse sampling model of RBSR as an underdetermined equation-solving problem, the received signals are sparsely recovered in target domain. Two algorithms, including smooth approximation algorithm and focal underdetermined system solver (FOCUSS), based on different optimization ideas, are adopted to solve the problem. Simulation results show that compressive sampling methods can recover the target domain accurately, especially under the condition of high signal-to-noise ratio (SNR).
\end{abstract}

Keywords: Sparse sampling; Angular superresolution; Sparse signal reconstruction; Real beam scanning radar

\section{Introduction}

Real beam scanning radar (RBSR) has been widely used in both civilian and military fields, owing to its all weather and day/night ability. However, it is hard to realize the high azimuth resolution of stationary platform or irregular motion platform by traditional signal processing algorithms, such as matched filtering or Doppler beamforming (DBF) $[1,2]$. Although, we can obtain a high-range resolution by transmitting high bandwidth linear FM signal and using the matched filtering technique [3], the limited azimuth angular resolution and unmatched twodimensional radar image greatly restrict the application of the RBSR system. In order to obtain a high azimuth resolution, angular superresolution methods, including deconvolution and shift-and-convolution were proposed $[4,5]$. However, the deconvolution method is sensitive to noise and a large number of azimuth sampling points are needed to guarantee the superresolution performance. The shiftand-convolution technique, which is based on specific assumptions, also needs very large number of sampling

\footnotetext{
*Correspondence: levin1110@163.com

${ }^{\dagger}$ Equal contributors

College of Electronic Engineering, University of Electronic Science and

Technology of China, Xiyuan Ave, Chengdu 611731, China
}

data. Both of the above techniques greatly increase the computational efficiency of processing module.

In recent years, sparsity-based techniques were proposed and have been used in spectral estimation, image processing, and radar imaging [6-17]. In [6-9], a kind of novel DOA estimation methods based on the sparse signal recovery were proposed, which took advantage of the sparse distribution of azimuth target when the number of sampling data is limited. Compared with traditional DOA estimation algorithms, these algorithms are less sensitive to initialization and SNR, with less snapshots. M Elad et al. also introduced sparse and redundant representations to signal and image processing [10-12], such as wavelet denoising, image reconstruction and restoration and feature selection in machine learning. In addition, $\mathrm{Q}$ Liang et al. applied the compressive sensing technique to synthetic aperture radar (SAR) and radar sensor networks to tremendously reduce the sampling rate [13-17].

However, little work was reported on sparse signal recovery for the real beam system. Most of the previous studies concentrate on the improvement of performance and robustness of traditional superresolution algorithms. Recently, according to the similar signal properties of real beam scanning model and array signal model, several spectrum estimation methods are used to realize angular

\section{是 Springer}

(c) 2014 Zhang et al:- licensee Springer. This is an Open Access article distributed under the terms of the Creative Commons Attribution License (http://creativecommons.org/licenses/by/4.0), which permits unrestricted use, distribution, and reproduction in any medium, provided the original work is properly credited. 
superresolution $[18,19]$. Sparse signal recovery theory of array signal processing is a mature mathematics theory. So it is possible to establish the real beam sparse signal model by greatly increasing the sampling interval, and realize angular superresolution imaging by utilizing sparse signal recovery algorithms.

In the references of sparse signal reconstruction for array signal processing, $L_{1}$ norm is often used to express the sparsity. The smooth approximation algorithm is a classic sparse signal recovery algorithm which is based on the constraint of $L_{1}$ norm [20]. Other efficient signal recovery algorithms based on different constraints are also proposed, such as the focal underdetermined system solver (FOCUSS) algorithm [21,22]. In this paper, a real beam signal model is built first by sparsely sampling in the azimuth dimension, introduce the smooth approximation algorithm and the FOCUSS algorithm are introduced to deal with the underdetermined system as a comparison to verify the feasibility of angular superresolution for sparse sampling model of the real beam radar. The distribution of target and noise is not needed, and the range resolution is not restricted in these methods. Furthermore, the burden and cost of the radar system can be significantly reduced.

This paper is outlined as follows: The sparse sampling model of RBSR is given in the next section. The objective functions based on sparse constrains are built and solved by smooth approximation technique and FOCUSS algorithm in section 'Real beam smooth approximation algorithm' and section 'Real beam FOCUSS algorithm', respectively. The simulation results are provided to illustrate the performances of the two algorithms in the section that follows. The last section concludes this paper.

\section{Sparse representation of signal model}

Figure 1 shows the sampling model of RBSR. In this model, the antenna illuminates the detection region step by step with velocity $\omega$. The antenna transmits signal with uniform pulse repetition interval (PRI). The beamwidth of antenna is $\theta_{\text {beta }}$. Suppose that the azimuth scanning region is fixed, the scanning region of azimuth dimension can be discretized as a $M \times 1$ target amplitude vector of $\mathbf{x}$. In real beam radar imaging, large dimension of $\mathbf{x}$ implies more available angles and azimuth amplitude information, so large dimension of $\mathbf{x}$ is needed.

Because the imaging process of RBSR can be regarded as the convolution of antenna pattern function and discretized target vector, the signal model can be written as the following matrix form

$$
\mathbf{y}=\mathbf{A x}+\mathbf{n}
$$

In conventional convolution model, the received sampling signal $\mathbf{y}$ must have the same dimension as $\mathbf{x}$ to ensure that (1) is resolvable by traditional deconvolution methods. So $\mathbf{y}$ is an $M \times 1$ received signal vector, $\mathbf{x}$ is an $M \times 1$ target amplitude vector, $\mathbf{n}$ is an $M \times 1$ noise vector, convolution matrix $\mathbf{A}=\left[\mathbf{a}_{1}, \mathbf{a}_{2}, \cdots, \mathbf{a}_{M}\right]$ is an $M \times M$ matrix. For stationary platform, the offset of antenna and target in

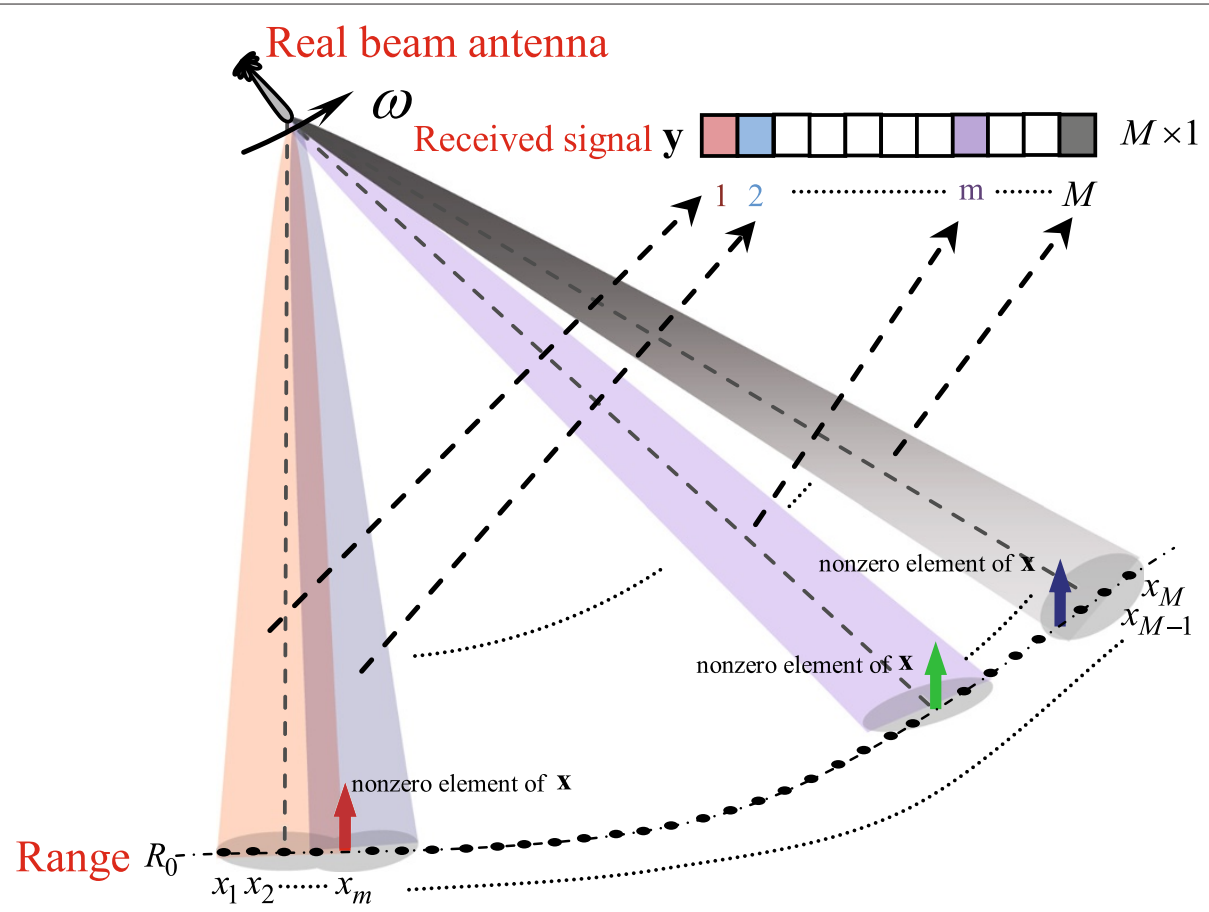

Figure 1 Sampling model of real beam scanning radar. 
the same range unit are all the same. It is not needed to consider the influence of phase. For irregular motion platform, the offset of antenna and target can be eliminated by motion compensation. So vector $\mathbf{a}_{m}$ in matrix $\mathbf{A}$ can be written as the real value

$$
\mathbf{a}_{m}=[\underbrace{0, \cdots, 0}_{m-1}, a_{1}, \cdots, a_{N}, 0, \cdots, 0]^{T}
$$

where $a_{1}, \cdots, a_{N}$ is the normalized antenna pattern sequence and $a_{n}$ is the normalized weighting of antenna pattern at corresponding position, $N=\theta_{\text {beta }} /(\omega \cdot \mathrm{PRI})$.

In RBSR imaging, in order to realize the high azimuth resolution, a large number of sampling signal are required in traditional superresolution methods; even $\mathbf{x}$ is sparse. But if $\mathbf{x}$ is $K$ sparse, $K \ll M$, and most of the element in $\mathbf{X}$ are zero, we can establish the sparse sampling signal model of RBSR. The requirement of sampling signal number will be significantly reduced when sparse signal recovery methods are used to solve this angular superresolution problem.

In the sparse sampling signal model of RBSR, the dimension of vector $\mathbf{x}$ is still $M \times 1$. The received signal is obtained by sparse sampling the imaging region. The number of received signal $\mathbf{y}$ can be calculated by $\Delta \phi /(\omega \cdot \mathrm{PRI})$, where $\Delta \phi=\phi_{2}-\phi_{1}$ is the scanning range, $\phi_{1}$ and $\phi_{2}$ express the initial and termination incidence angles of scanning region, respectively. For fixed scene, the number of sampling received signal is determined by $\omega$ and PRI. So the form of Equation 1 is equivalent to the basic version of sparse representation problem with noise when we significant increase $\omega$ or PRI to make the dimension of vector $\mathbf{y}$ much less than $M \times 1$. Furthermore, all the following discussion in this paper are based on the assumption that $\mathbf{x}$ is sparse, and the dimension of $\mathbf{y}$ is much less than $\mathbf{x}$ in (1). Therefore, the angular superresolution problem is to find a sparse $\mathbf{x} \in C^{M}$ of Equation 1.

The problem of Equation 1 is ill-posed and has infinitely many solutions. The best choice to express the sparsity of nonzero entries in $\mathbf{x}$ is $L_{0}$ norm, which makes the problem a NP-hard combinatory optimization problem and very difficult to solve. One of the most common method is replace $L_{0}$ norm with $L_{1}$ norm $[23,24]$. It denoted by $\|\mathbf{x}\|_{1}^{1}$ at mathematical. So the form for $L_{1}$ norm approximation problem is

$$
\min \|\mathbf{y}-\mathbf{A x}\|_{2}^{2}+\lambda\|\mathbf{x}\|_{1}
$$

The first term of (3) forces the residual error to be small, and the $L_{1}$-term enforces the sparsity of target domain. $\lambda$ is the parameter which controls the tradeoff between the residual error and sparsity of target domain. Some wellknown ideas, such as the discrepancy principle and the L-curve have been proposed to select $\lambda[25,26]$. So the problem of how to choose appropriate $\lambda$ is not discussed in this paper. In addition, to guarantee the sparse characteristic of $\mathbf{x}$, the $L_{1}$ norm also makes the problem as a convex optimization problem. In this case, the global optimum can be found. This objective function has been used in many real-valued and complex-valued data sparse signal representation works.

In the RBSR signal model, the convolution matrix has lots of zero elements which is different from the steering matrix in array signal model. So, we introduce the smooth approximation technique to solve Equation 3 and demonstrate the feasibility of sparse angular superresolution of RBSR. Furthermore, Equation 4 is not the only formed to express the sparsity of $\mathbf{x}$, FOCUSS algorithm is another typical sparse signal recovery method based on the minimum norm least square frame. The re-weighted matrix is used to alter the weight of different targets in objective function, which makes true targets more prominent. We also introduce this algorithm in the sparse signal recovery problem of RBSR.

\section{Superresolution by sparse signal reconstruction}

In this section, the smooth approximation technique and FOCUSS algorithm are introduced to the sparse sampling model of RBSR and realized sparse signal reconstruction.

\section{Real beam smooth approximation algorithm}

In this subsection, we develop the smooth approximation algorithm for the underdetermined formation based on a regularized target reconstruction framework. The smooth approximation technique is an effective method which makes objective function solvable by smooth approximation of $L_{1}$ norm [20,27]. The method provides an extension of real-valued feature-preserving image reconstruction methods, to the angular superresolution problem. By applying this algorithm to real beam scanning imaging, only a few sampling data is needed to improve the resolution of point target and the interference of noise and clutter is reduced.

Because of $L 1$ norm is non-differentiable at original point, it is difficult to directly calculate the derivative of Equation 3. This algorithm utilizes the smooth approximation technique, solving the derivative problem of $L 1$ norm. The following slightly modified cost function was used to replace Equation 3

$$
J(\mathbf{x})=\|\mathbf{y}-\mathbf{A} \mathbf{x}\|_{2}^{2}+\lambda \sum_{k=1}^{K}\left(\left(f_{k}\right)^{2}+\varepsilon\right)^{1 / 2}
$$

where $\varepsilon$ is a small constant which is larger than zero. Note that this equation is closed to Equation 4 when $\varepsilon$ is infinitely small. Then we calculate the gradient of 
Equation 4 with respect to $\mathbf{x}$. We can obtain the following expression

$$
\nabla J(\mathbf{x})=\mathbf{A}^{\mathrm{H}} \mathbf{A} \mathbf{x}-\mathbf{A}^{\mathrm{H}} \mathbf{y}+\lambda \Lambda(\mathbf{x}) \mathbf{x}
$$

where $\Lambda(\mathbf{x})=\operatorname{diag}\left\{\left(\left|x_{k}\right|^{2}+\varepsilon\right)^{-\frac{1}{2}}\right\}$. Then we build a new Hessian update scheme by extending ideas from halfquadratic regularization to the signal recovery problem of RBSR. The gradient expression of (5) can be rewritten as

$$
\nabla J(\mathbf{x})=\left(\mathbf{A}^{\mathrm{H}} \mathbf{A}+\lambda \Lambda(\mathbf{x})\right) \mathbf{x}-\mathbf{A}^{\mathrm{H}} \mathbf{y}=\mathbf{Q}(\mathbf{x}) \mathbf{x}-\mathbf{A}^{\mathrm{H}} \mathbf{y}
$$

In expression (6), we can use $\mathbf{Q}(\mathbf{x})$ as an approximation to the Hessian. So we use this approximate Hessian $\mathbf{Q}(\mathbf{x})$ in the quasi-Newton iteration

$$
\hat{\mathbf{x}}^{(i+1)}=\hat{\mathbf{x}}^{(i)}-\gamma\left[\left(\mathbf{A}^{\mathrm{H}} \mathbf{A}+\lambda \Lambda\left(\hat{\mathbf{x}}^{(i)}\right)\right)\right]^{-1} \nabla J\left(\hat{\mathbf{x}}^{(i)}\right)
$$

where $\gamma$ is the step size. After substituting (6) into (7), we obtain the iterative expression

$$
\begin{aligned}
\left(\mathbf{A}^{\mathrm{H}} \mathbf{A}+\lambda \Lambda\left(\hat{\mathbf{x}}^{(i)}\right)\right) \hat{\mathbf{x}}^{(i+1)}= & (1-\gamma)\left(\mathbf{A}^{\mathrm{H}} \mathbf{A}\right. \\
& \left.+\lambda \Lambda\left(\hat{\mathbf{x}}^{(i)}\right)\right) \hat{\mathbf{x}}^{(i)}+\gamma \mathbf{A}^{\mathrm{H}} \mathbf{y}
\end{aligned}
$$

In addition, another small constant value $\delta$ which is also larger than zero was taken to determine the terminating condition of iteration. The iteration will stop when $\left\|\hat{\mathbf{x}}^{(i+1)}-\hat{\mathbf{x}}^{(i)}\right\|_{2}^{2} /\left\|\hat{\mathbf{x}}^{(i)}\right\|_{2}^{2} \leq \delta$. We can select appropriate $\delta$ according to the requirement of superresolution performance or efficiency.

\section{Real beam FOCUSS algorithm}

The FOCUSS algorithm is introduced to solve the angular superresolution problem in this subsection. The essence of FOCUSS algorithm is the weighted minimum norm least square method (MNLS). The iterations are based on weighted norm minimization of the dependent variable with the weights being a function of the preceding iterative solutions $[21,22]$. The algorithm obtains the unique and sparse solution from underdetermined equations, which has been already used for DOA estimation and neuromagnetic imaging problem. By applying this algorithm to the superresolution problem of RBSR from sparse sampling data, we can obtain the high-resolution results in theory. The basic weighted least square form is

$$
\min \|\mathbf{y}-\mathbf{A x}\|_{2}^{2}+\left\|\mathbf{W}^{-1} \mathbf{x}\right\|_{2}^{2}
$$

where $\mathbf{W}$ is the $K \times K$ weighted diagonal matrix which controls the choice of estimation vector. We can obtain the weighted least square solution by solving Equation 9

$$
\hat{\mathbf{x}}=\mathbf{W} \mathbf{W}^{\mathrm{H}} \mathbf{A}^{\mathrm{H}}\left(\mathbf{A} \mathbf{W} \mathbf{W}^{\mathrm{H}} \mathbf{A}^{\mathrm{H}}\right)^{-1} \mathbf{y}
$$

where the weighted diagonal matrix is $\mathbf{W}=\operatorname{diag}$ $(x(1), \ldots, x(K))$. Because the diagonal matrix can be reconstructed by the calculation results of Equation 10, we can build the iterative expression

$$
\hat{\mathbf{x}}_{k}=\mathbf{W}_{k} \mathbf{W}_{k}^{\mathrm{H}} \mathbf{A}^{\mathrm{H}}\left(\mathbf{A} \mathbf{W}_{k} \mathbf{W}_{k}^{\mathrm{H}} \mathbf{A}^{\mathrm{H}}\right)^{-1} \mathbf{y}
$$

where $\mathbf{W}_{k}=\operatorname{diag}\left(x_{k-1}(1), \ldots, x_{k-1}(K)\right)$. From the iterative expression, we can find that the diagonal matrix is $a$ posteriori weight in iterations. In each iterative step, the term of $\left\|\mathbf{W}^{-1} \mathbf{x}\right\|_{2}^{2}$ reduces the weight of strong scatter targets, which make them stronger than weak scatter points and noise in each steps of iterations. It is easily proven that the nonzero term in estimation result $\hat{\mathbf{x}}$ is smaller than vector dimension $K$, so the solution is sparse.

Besides, because the computed results are affected by the iterative initialization, it is necessary to select an appropriate one. In the references, the Moore-Penrose pseudo-inverse matrix is used to calculate the iterative initialization. The calculation method of pseudo-inverse matrix is based on the least square frame, $\min \|\mathbf{y}-\mathbf{H x}\|_{2}^{2}$. The solution can be written as

$$
\hat{\mathbf{x}}=\mathbf{A}^{\mathrm{H}}\left(\mathbf{A A}^{\mathrm{H}}\right)^{-1} \mathbf{y}=\mathbf{A}^{\#} \mathbf{y}
$$

where $\mathbf{A}^{\#}=\mathbf{A}^{\mathrm{H}}\left(\mathbf{A A}^{\mathrm{H}}\right)^{-1}$ is the Moore-Penrose pseudoinverse matrix. However, in low signal-to-noise ratio (SNR) condition, the coarse estimation results by MoorePenrose pseudo-inverse matrix have huge estimation errors to the true target distribution, which will introduce the large estimate deviations of target location and amplitude. So this paper utilizes the simple method to obtain a rather coarse, but little estimate deviation of $\hat{\mathbf{x}}$. The estimation result is obtained by employing a matched filter bank strategy which can be written as

$$
\hat{\mathbf{x}}=\mathbf{A}^{\mathrm{H}} \mathbf{y}
$$

Otherwise, this algorithm uses the same method to select iterations as in section 'Real beam smooth approximation algorithm'.

\section{Simulations and discussions}

In this section, angular superresolution is performed by the two algorithms that have been presented in section 'Superresolution by sparse signal reconstruction'. Several simulation using sparse sampling data were taken to illustrate the potential of sparse signal recovery for the issue of high angular resolution of RBSR. Consider three targets with the same amplitude which were located at $-4^{\circ}, 2^{\circ}$ and $3.5^{\circ}$, respectively. The scanning region of single real beam antenna is from $-8^{\circ}$ to $8^{\circ}$, and the dimension of the underdetermined system $\mathbf{A}$ is $25 \times 500$. Simulation results are shown in the following figures. 

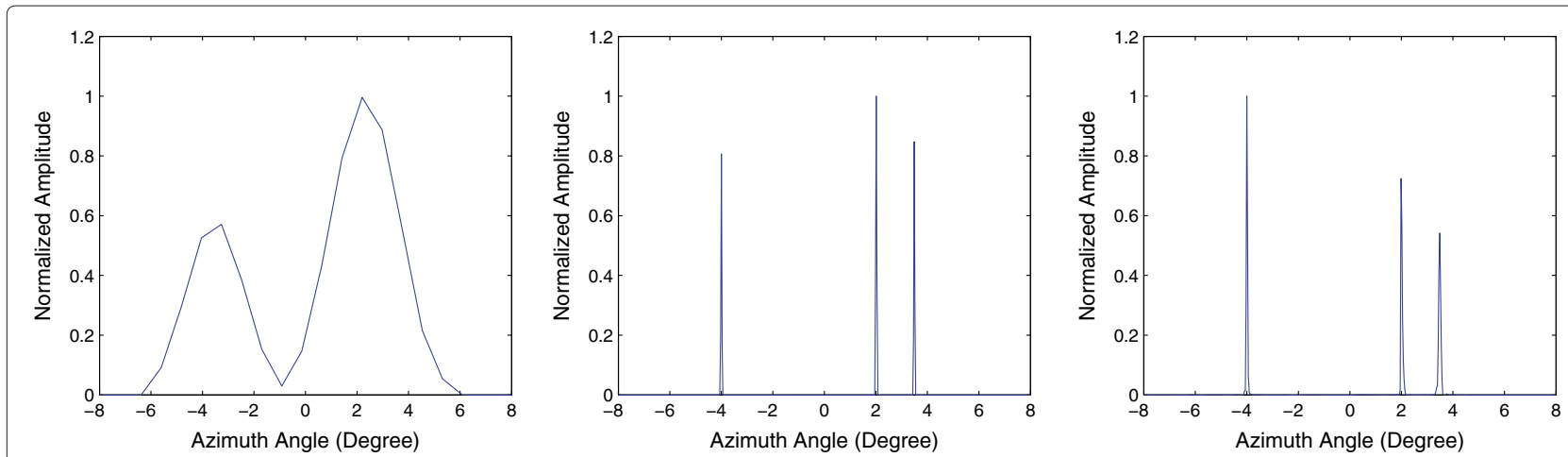

Figure 2 Simulation results of the two algorithms in ideal environment. (a) Real beam sparse sampling echo; (b) Simulation result of smooth approximation technique; (c) Simulation result of FOCUSS algorithm.

In Figure 2, the received echo is noiseless. The recovery results of the two algorithms are obtained with optimal iterations 8 and 50, respectively. In this case, both of the algorithms recovered the target domain successfully. The feasibility of the sparse signal recovery methods of the sparse sampling model of RBSR were demonstrated in these results. However, the FOCUSS algorithm is more efficient than the smooth approximation technique in ideal condition in consideration of the iterations. To determine the robustness of the two algorithms, the recovery results of the received echo with noise are given in Figures 3 and 4. The additional white noise obeys the Gaussian distribution with variance $\sigma^{2}$ which is determined from the specified SNR level as

$$
\sigma^{2}=\frac{1}{M}\|\mathbf{A x}\|^{2} 10^{-S N R / 10}
$$

Figures 3 and 4 show the signal recovery results of smooth approximation technique and FOUCSS algorithm. The SNR of Figures 3 and 4 are 20 and $10 \mathrm{~dB}$, respectively. In simulation, the value of $\mathbf{A x}$ which was used to calculate variance $\sigma^{2}$ of additional noise is the actual

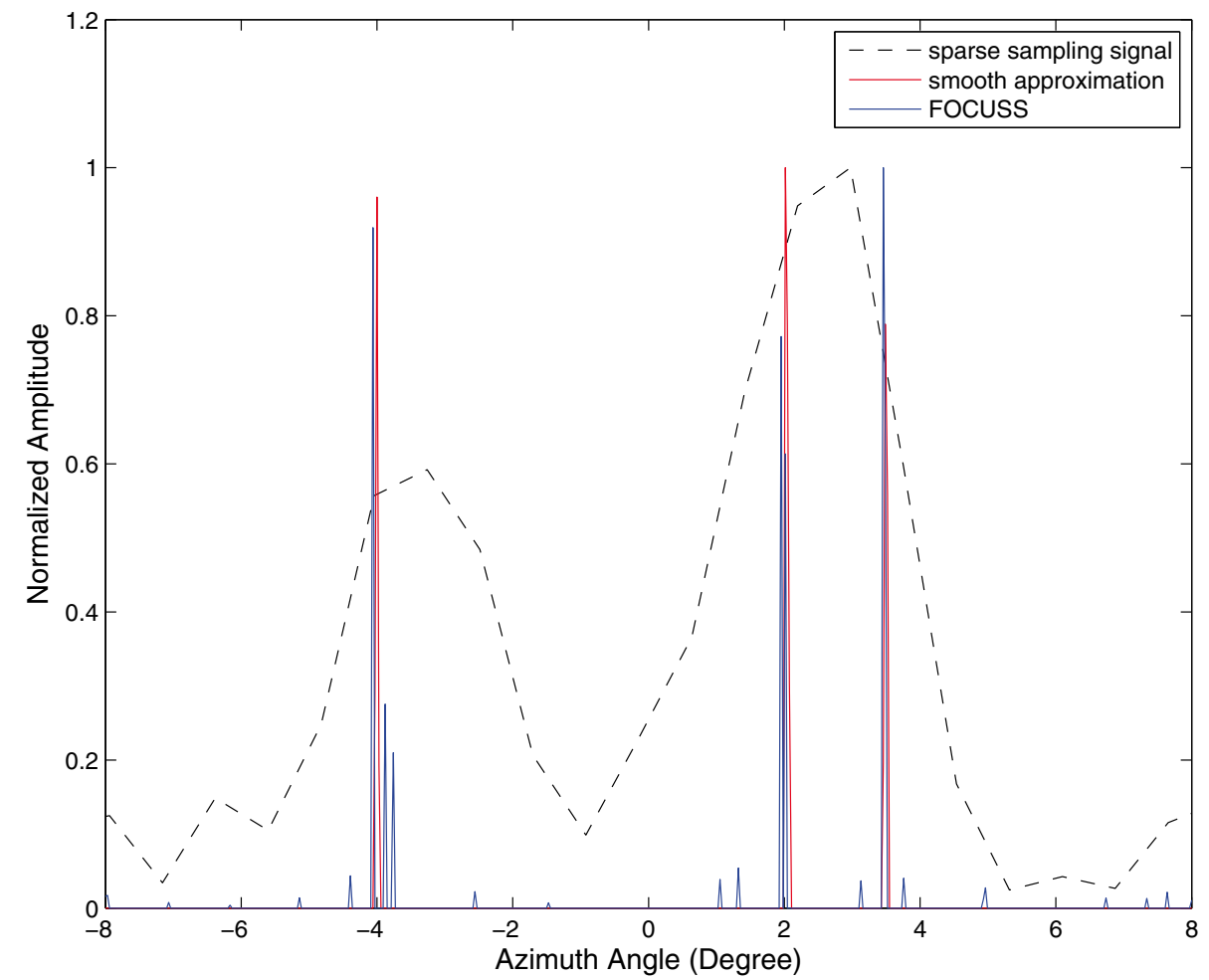

Figure 3 Simulation results of the two algorithms with a $20 \mathrm{~dB}$ SNR. The dotted line is the real beam sparse sampling echo, the red and blue lines express the simulation results of smooth approximation technique and FOCUSS algorithm, respectively. 


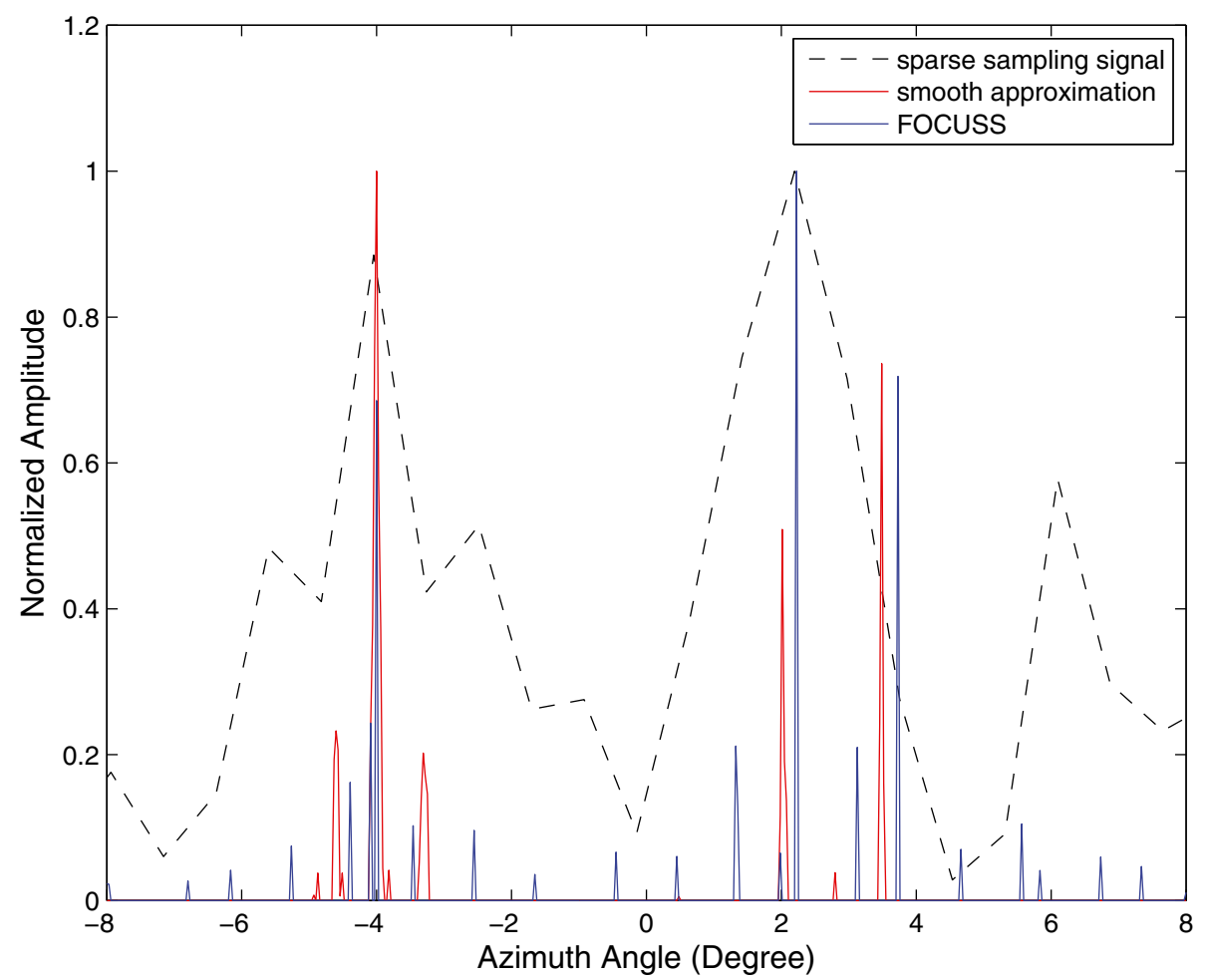

Figure 4 Simulation results of the two algorithms with a 10 dB SNR. The dotted line is the real beam sparse sampling echo, the red and blue lines express the simulation results of smooth approximation technique and FOCUSS algorithm, respectively.

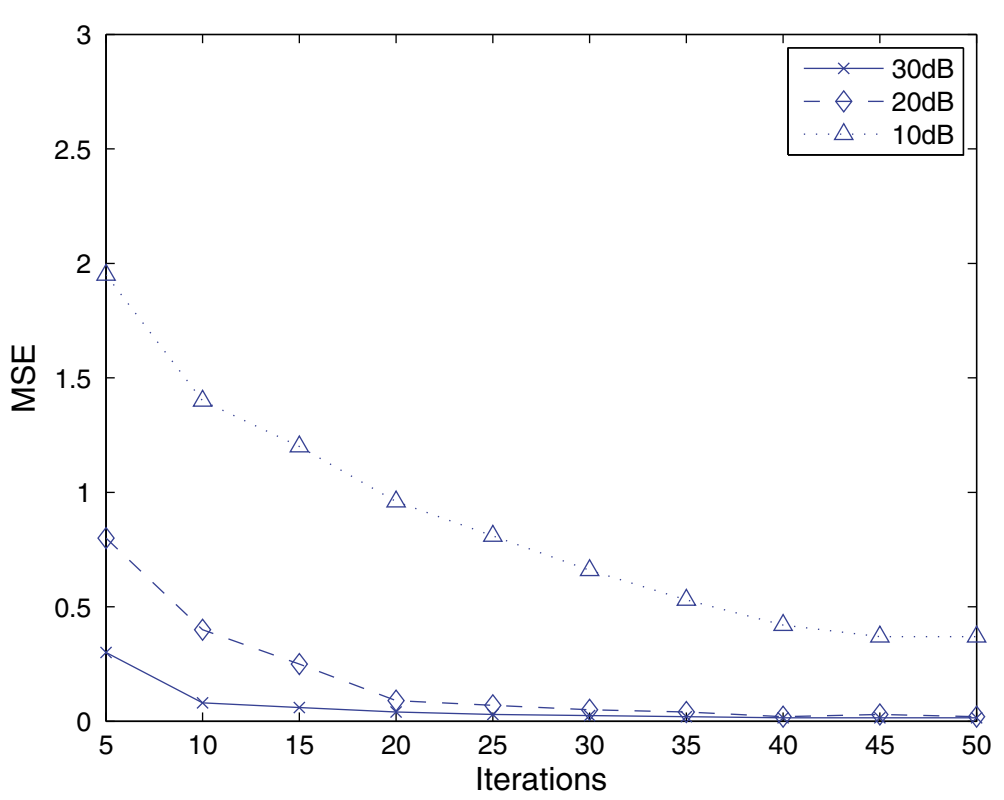

Figure 5 MSE curves of smooth approximation technique in different SNR conditions. Horizontal and vertical coordinates express iterations and MSE, respectively. The curves express the regularity of MSE in different SNR conditions. 


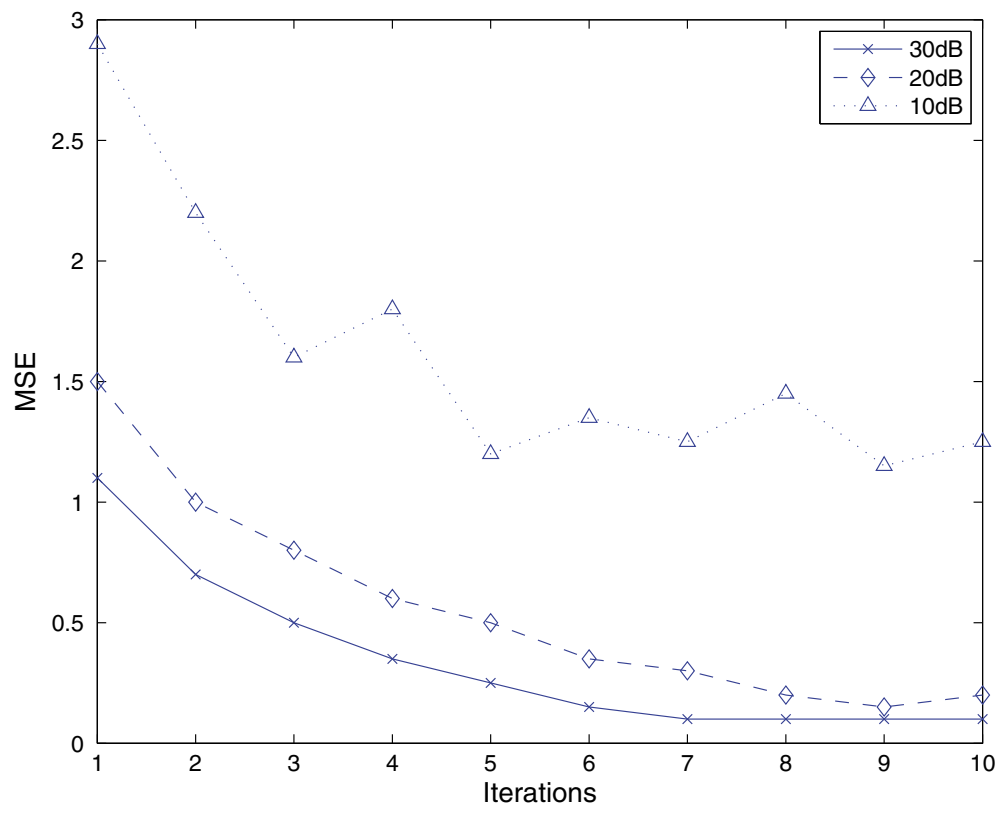

Figure 6 MSE curves of FOCUSS in different SNR condition. Horizontal and vertical coordinates express iterations and MSE, respectively. The curves express the regularity of MSE in different SNR condition.

value. Then we used the absolute value of vector $\mathbf{y}$ to recover the target amplitude vector. Simulation results in Figures 3 and 4 are normalized to clearly show the performance of two algorithms. From the two figures, we can see that the smooth approximation technique has better performance in low SNR condition. Little spurious peaks and small changes of amplitude and location of target appeared in the simulation results of the smooth approximation technique. However, more spurious peaks appear in the results of the FOCUSS algorithm. The amount and amplitude of spurious peaks increased with the increase of noise intensity. The processing result of FOCUSS algorithm has reached an unacceptable level when the SNR is $10 \mathrm{~dB}$.

Figures 5 and 6 show the superresolution performance of the two algorithms for various choices of SNR in terms of mean squared error (MSE). In this paper, MSE is calculated as

$$
\operatorname{MSE}=E\left(\frac{\|\hat{\mathbf{x}}-\mathbf{x}\|_{2}^{2}}{\|\mathbf{x}\|_{2}^{2}}\right)
$$

where $\hat{\mathbf{x}}$ and $\mathbf{x}$ are the estimated and true solution. A comparison of the performance curves indicates that the FOCUSS algorithm has better convergence speed. But compared with smooth approximation technique, the MSE of FOCUSS algorithm is not only larger and but also increased more rapidly with the increase of noise intensity under the same SNR. It is shown in Figure 6 that low SNR leads to degradation of the estimated performance of the FOCUSS algorithm. The smooth approximation technique has a better property of convergence, it still works well when the SNR is $10 \mathrm{~dB}$.

\section{Conclusion}

In traditional angular superresolution algorithms of real beam scanning radar, large amounts of received data is necessary. However, the requirement of data increase the complex of hardware platform. Compressive sensing theory can reduce the requirement of sample rate and length of received data while guarantee the superresolution performance. The sparse signal recovery technique could exactly reconstruct the target domain from sparse sampling signal of RBSR.

This paper studies real beam angular superresolution problem based on the sparse signal reconstruction technique. Firstly, the sparse sampling signal model of the RBSR system is built as a sparse representation form. Then, two sparse signal recovery techniques are chosen to realize signal reconstruction in the target domain. Finally, simulations are given to show that both of the two methods can effectively improve the angular resolution, and the superresolution effectiveness are affected by SNR of received signal somewhat. The next step in our research work will consist in improving algorithm that reduces the effect of noise.

\section{Competing interests}

The authors declare that they have no competing interests.

\section{Acknowledgements}

This work was supported by the National Natural Science Foundation of China (Grant No.B1420110182), and the Pre-Research (Grant No.402040202). 
Received: 28 February 2014 Accepted: 2 July 2014

Published: 12 July 2014

\section{References}

1. CY Kit, KV Chet, An introduction to synthetic aperture radar (SAR). Prog Electromagnetics Res. B. 2, 27-60 (2008)

2. L Teng, $L$ Zheng, $Z$ Ding, $A$ DBS Doppler centroid estimation algorithm based on entropy minimization. Geosci. Remote Sensing IEEE Trans. 49, 3703-3712 (2011)

3. DR Wehner, High resolution radar. (MA, Artech, House, Inc, Norwood, 1987)

4. J Guan, Y Huang, J Yang, Improving angular resolution based on maximum a posteriori criterion for scanning radar, in Radar Conference (RADAR) (Atlanta, GA, 7-11 May 2012, 2012), pp. 0451-0454

5. F Perez-Martinez, J Garcia-Fominaya, J Calvo-Gallego, A shift-and-convolution technique for high-resolution radar images. Sensors J. IEEE. 5, 1090-1098 (2005)

6. Fuchs J, On the application of the global matched filter to DOA estimation with uniform circular arrays. Signal Process. IEEE Trans. 49, 702-709 (2001)

7. D Malioutov, M Cetin, A Willsky, A sparse signal reconstruction perspective for source localization with sensor arrays. Signal Process. IEEE Trans. 53, 3010-3022 (2005)

8. S Petre, B Prabhu, L Jian, Spice: a sparse covariance-based estimation method for array processing. Signal Process. IEEE Trans. 59, 629-638 (2011)

9. I Bilik, Spatial compressive sensing for direction-of-arrival estimation of multiple sources using dynamic sensor arrays. Aerosp. Electron. Syst. IEEE Trans. 47, 1754-1769 (2011)

10. M Elad, Sparse and redundant representations: from theory to applications in signal and image processing. (Springer, New York, 2010)

11. S Sardy, P Tseng, A Bruce, Robust wavelet denoising. Signal Process. IEEE Trans. 49, 1146-1152 (2001)

12. J Starck, F Murtagh, J Fadili, Sparse image and signal processing: wavelets, curvelets, morphological Diversity. (Cambridge University Press, New York, NY, 2010)

13. Q Liang, Compressive sensing for synthetic aperture radar in fast-time and slow-time domains, in 2011 Conference Record of the Forty Fifth Asilomar Conference on Signals, Systems and Computers (ASILOMAR) (Pacific Grove, CA, 6-9 Nov 2011), pp. 1479-1483

14. Q Wu, Q Liang, Coprime sampling for nonstationary signal in radar signa processing. EURASIP J. Wireless Commun. Netw. 2013(1), 1-11 (2013)

15. J Chen, Q Liang, B Zhang, Spectrum efficiency of nested sparse sampling and coprime sampling. EURASIP J. Wireless Commun. Netw. 2013(1), $1-15$ (2013)

16. Q Liang, Compressive sensing for radar sensor networks, in Global Telecommunications Conference (GLOBECOM) (Miami, FL, 6-10 Dec 2010), pp. 1-5

17. Q Liang, J Wu, X Cheng, Sparsity and compressive sensing of sense-through-foliage radar signals. 2012 IEEE International Conference on Communications (ICC), 6376-6380 (2012)

18. S Uttam, N Goodman, Superresolution of coherent sources in real-beam data. Aerosp. Electron. Syst. IEEE Trans. 46(3), 1557-1566 (2010)

19. Y Zhang, Y Zhang, W Li, Y Huang, Y Huang, J Yang, Angular superresolution for real beam radar with iterative adaptive approach, in 2013 IEEE International Geoscience and Remote Sensing Symposium (IGARSS) (Melbourne VIC, 21-26 July 2013), pp. 3100-3103

20. M Cetin, W Karl, Feature-enhanced synthetic aperture radar image formation based on nonquadratic regularization. Image Process. IEEE Trans. 10, 623-631 (2001)

21. I Gorodnitsky, B Rao, Sparse signal reconstruction from limited data using FOCUSS: a re-weighted minimum norm algorithm. Signal Process. IEEE Trans. 45, 600-616 (1997)

22. S Cotter, B Rao, K Engan, Sparse solutions to linear inverse problems with multiple measurement vectors. Signal Process. IEEE Trans. 53, 2477-2488 (2005)

23. SS Chen, DL Donoho, MA Saunders, Atomic decomposition by basis pursuit. SIAM J. Sci. Comput. 20, 33-61 (1998)

24. DL Donoho, For most large underdetermined systems of linear equations the minimal $\ell^{1}$-norm solution is also the sparsest solution. Commun. Pure Appl. Math. 59, 797-829 (2006)
25. C David, P Michele, P Roland, A simple method using Morozov's discrepancy principle for solving inverse scattering problems. Inverse Probl. 13, 1477 (1997)

26. PC Hansen, Analysis of discrete ill-posed problems by means of the L-curve. SIAM Rev. 34, 561-580 (1992)

27. C Pierre, B Laure, A Gilles, B Michel, Deterministic edge-preserving regularization in computed imaging. Image Process. IEEE Trans. 6. 298-311 (1997)

doi:10.1186/1687-6180-2014-110

Cite this article as: Zhang et al:: A sparse sampling strategy for angular superresolution of real beam scanning radar. EURASIP Journal on Advances in Signal Processing 2014 2014:110.

\section{Submit your manuscript to a SpringerOpen ${ }^{\circ}$ journal and benefit from:}

- Convenient online submission

- Rigorous peer review

- Immediate publication on acceptance

- Open access: articles freely available online

- High visibility within the field

- Retaining the copyright to your article

Submit your next manuscript at $\boldsymbol{\nabla}$ springeropen.com 